\title{
SELETIVIDADE DE HERBICIDAS NA CULTURA DO CÁRTAMO
}

\section{HERBICIDE SELECTIVITY IN SAFFLOWER CROP}

Fábio Henrique Krenchinski ${ }^{\mathrm{a}}$, Vinicius Gabriel Caneppele Pereira ${ }^{\mathrm{a}}$, Bruno Flaibam Giovanelli ${ }^{\mathrm{a}}$, Mateus Dalpubel Mattiuzzi ${ }^{\mathrm{b}}$, Alfredo Junior Paiola Albrecht ${ }^{\mathrm{c}}$, Felipe Gustavo Wagner ${ }^{\mathrm{d}}$

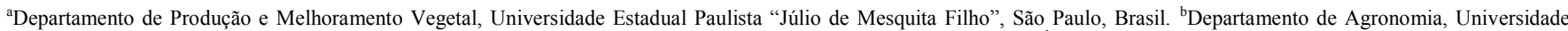
Estadual de Maringá, Paraná, Brasil. ' Departamento de Ciências Agronômicas, Universidade Federal do Paraná, Brasil. ${ }^{\mathrm{d}}$ Tropical Melhoramento \& Genética, Paraná, Brasil.

*Autor correspondente: bfgiovanelli@yahoo.com.br

\section{INFORMAÇÕES DO ARTIGO \\ Histórico do artigo:}

Recebido: 09 Outubo 2018.

Aceito: 03 Abril 2019.

Publicado: 08 Agosto 2019.

\section{Palavras-chave/Keywords:}

Carthamus tinctorius L./ Carthamus tinctorius L.,

Controle químico/ Chemical control.

Pós-emergência/ Incorporated preplanting.

Pré-plantio/ Post-emergence.

Pré-plantio incorporado/ Pre-planting.

\section{Financiamento:}

Universidade Federal do Paraná (UFPR).

Direito Autoral: Este é um artigo de acesso aberto distribuído sob os termos da Licença Creative Commons, que permite uso, distribuição e reprodução irrestritos em qualquer meio, desde que $\mathrm{o}$ autor e a fonte originais sejam creditados.

\section{Citação deste artigo:}

KRENCHINSKI, F. H.; PEREIRA, V. G. C.; GIOVANELLI, B. F.; MATTIUZZI, M. D.; ALBRECHT, A. J. P.; WAGNER, F. G. Seletividade de herbicidas na cultura do cártamo. Revista Brasileira de Herbicidas, v. 18, n.1. 2019.

\begin{abstract}
RESUMO
O cártamo (Carthamus tinctorius L.) é uma planta oleaginosa que apresenta elevado potencial de cultivo no Brasil. O controle das plantas daninhas na cultura é essencial para proteger o seu potencial produtivo. O objetivo deste estudo foi avaliar a seletividade de herbicidas aplicados em pré-plantio incorporado (PPI), pré-plantio (PRÉ) e pós-emergência (PÓS) do cártamo. Ao todo, três estudos foram instalados em casa de vegetação, em delineamento inteiramente casualizado, com cinco repetições. Os herbicidas avaliados para aplicação em PPI (Experimento I) foram: diclosulam, metribuzin, pendimethalin e trifluralin; para aplicação em PRÉ (Experimento II) foram utilizados os herbicidas: 2,4-D, ametryn+clomazone, atrazine, carfentrazone, carfentrazone + sulfrentrazone, clomazone, diclosulam, flumioxazin, imazaqin, imazethapyr, isoxaflutole, lactofen, mesotrione, metribuzin, metsulfuron - methyl, pendimethalin, saflufenacil, s-metolachlor, sulfrentrazone e trifluralin e, para a aplicação em PÓS (Experimento III) foram avaliados: ametryn+clomazone, atrazine, bentazon, bentazon + imazamox, carfentrazone, chlorimuron, clethodim + fenoxaprop, clethodim, clodinafop, floroxypyr, fluazifop+fomesafen, fluazifopP, flumiclorac-pentyl, flumioxazin, fluroxipir, fomesafen, foramsulfuron + iodosulfuron, haloxyfop, imazethapyr, iodosulfuron, lactofen, mesotrione, nicosulfuron, tembotrione e tepraloxydim), comparados com a testemunha sem aplicação. A fitotoxicidade foi avaliada aos 7, 14, 21 e 28 dias após aplicação (DAA), usando a escala percentual de zero a 100, e a massa fresca e seca foram obtidas aos 35 DAA. Os dados foram submetidos a análise de variância e, as médias dos tratamentos foram comparadas pelo teste de Tukey $(p \leq 0,05)$. O herbicida trifluralin apresenta seletividade a cultura do cártamo nas modalidades de PPI e PRÉ, enquanto que cletodim, fluazifop-p-butyl e haloxifope-p-metílico são seletivos para aplicação em PÓS.
\end{abstract}

\section{ABSTRACT}

Safflower (Carthamus tinctorius L.) is an annual oleaginous plant that presents high potential in Brazilian agriculture. The chemical control of weeds in the crop is essential to avoid damage and protect productive potential. The objective of this study was to evaluate the selectivity of herbicides applied in pre-planting incorporated (PPI), pre-planting (PRE) and post-emergence (POST) of the safflower crop. For all, three experiments were installed in greenhouse with a completely randomized design with five replications. The herbicides evaluated for application in PPI (Experiment I) were: diclosulam, metribuzin, pendimethalin and trifluralin; for the application in PRE (Experiment II) the herbicides were: 2,4-D, ametryn + clomazone, atrazine, carfentrazone, carfentrazone + sulfrentrazone, clomazone, diclosulam, flumioxazin, imazethapyr, isoxaflutole, lactofen, mesotrione, metribuzin, metsulfuron - ametryn + clomazone, atrazine, bentazon, bentazon + imazamox, carfentrazone, chlorimuron, clethodim + fenoxaprop, clethodim, clodinafop, were used for the application in POST (Experiment III), floroxypyr, fluazifop + fomesafen, fluazifop-P, flumiclorac-pentyl, flumioxazin, fluroxypyr, fomesafen, foramsulfuron + iodosulfuron, haloxyfop, imazethapyr, iodosulfuron, lactofen, mesotrione, nicosulfuron, tembotrione and tepraloxydim), compared to the control without herbicide application. The crop phytotoxicity was evaluated at 7, 14, 21 and 28 days after application (DAA), using the percentage scale from 0 to 100 , with fresh and dry mass obtained at 35 DAA. The data was submitted to analysis of variance and the means were compared by the Tukey test $(p \leq 0.05)$. Trifluralin herbicide is selective to the safflower crop in PPI and PRE aplications, while cletodim, fluazifop-p-butyl and haloxyfop-p-methyl are selective for application in POST. 


\section{Introdução}

O cártamo (Carthamus tinctorius L.) é uma planta oleaginosa pertencente à família Asteraceae que apresenta elevado potencial de ser utilizada em sistemas de cultivo no Brasil, devido a sua adaptação a diversas condições edafoclimáticas. A produtividade pode variar de 2 a 4 ton.ha $^{-1}$, e o seu teor de óleo varia de 20 a $40 \%$, dependendo do cultivar (FAOSTAT, 2019).

Dessa forma, apresenta elevado potencial na produção de biodiesel e alimentação humana (HAMMER ; HELLER, 1998), (RAHAMATALLA et al. 2001). A Ásia se apresenta como o maior continente produtor $(56,7 \%)$, e sua produção mundial em 2017 foi em torno de 690 mil toneladas (FAOSTAT, 2019). A planta possui considerável desenvolvimento em regiões com precipitações que variam de 300 a $600 \mathrm{~mm}$ anuais, tolerando uma alta amplitude de temperaturas $\left(-7\right.$ a $\left.40^{\circ} \mathrm{C}\right)$ (BAGHERI; SAM-DAILIRI, 2011).

O manejo de plantas daninhas na cultura do cártamo ainda é limitado, e é considerada uma etapa importante para proteção do cultivo, devido aos efeitos da competição por recursos do meio, como: água, luz, e nutrientes. Por se tratar de uma espécie de crescimento lento e ciclo mais longo, a competição com plantas daninhas é um fator mais limitante, podendo haver uma perda de produtividade de até $88 \%$, durante um Período Crítico de Prevenção à Interferência (PCPI) de 59 dias (MARQUES, 2017), ou seja, apresenta um período mais longo quando comparado ao de outras espécies como a soja, que pode variar de 33 a 42 dias após a emergência (NEPOMUCENO et al, 2007). Com isso, a utilização de herbicidas é um método de controle eficiente e necessário, podendo ser realizada em pré-plantio incorporado (PPI), pré-emergência (PRÉ) e pós-emergência (PÓS), devendo proporcionar vantagem competitiva à cultura (HARKER; O'DONOVAN, 2013).

Todavia, o controle de plantas daninhas através de herbicidas depende do uso de produtos seletivos à cultura, evitando a ocorrência de injúrias que possam afetar a produtividade (MARTINS et al. 2007). A seletividade depende de vários fatores, tanto fisiológicos, como edafoclimáticos, e também outros inerentes à espécie e ao herbicida (SILVA; SILVA, 2007). No entanto, é importante ressaltar que a avaliação de seletividade não consiste em apenas injúrias visuais, devido ao fato de algumas moléculas causarem injúrias, mas não alterarem a produtividade (NEGRISOLI et al. 2004).

Em outras culturas, como o crambe (Crambe maritima) (GRIGOLLI, 2015; SOUZA et al. 2014; DE OLIVEIRA NETO et al. 2011) e a mamona (Ricinus communis) (MACIEL et al. 2011), a seletividade de alguns herbicidas já é conhecida. Por sua vez, a cultura do cártamo não apresenta herbicidas registrados no Brasil e também há poucos produtos conhecidos que apresentem seletividade a cultura, assim como menos trabalhos a respeito do assunto na literatura, evidenciando a necessidade de mais estudos sobre a seletividade de herbicidas ao cártamo.

Portanto, o objetivo do presente trabalho foi avaliar a seletividade de herbicidas aplicados em pré-plantio incorporado, pré-emergência e pós-emergência da cultura do cártamo.

\section{Material e métodos}

A seletividade dos herbicidas $\mathrm{s}$ foi avaliada através de três experimentos (aplicação em PPI, PRÉ e PÓS) conduzidos em casa de vegetação, entre agosto e dezembro de 2015, utilizando o delineamento inteiramente casualizado, com cinco repetições. As plantas de cártamo foram alocadas em vasos plásticos (unidades experimentais - 1 planta por vaso) com capacidade para 5 litros, preenchido com solo coletado na camada de $0-20 \mathrm{~cm}$. Os dados da análise do solo evidenciaram os seguintes resultados: $\mathrm{pH}$ (Cacl2): 5,5; 5,39; 0,87; 0,51;0,00 e 11,05 $\mathrm{cmol}_{\mathrm{c}} \mathrm{dm}^{-3}$ de $\mathrm{Ca}, \mathrm{Mg}, \mathrm{K}, \mathrm{Al}$ e CTC, respectivamente, C: $13,65 \mathrm{~g} \mathrm{dm}^{-3}$; MO 23,48 $\mathrm{g} \mathrm{dm}^{-3}$; V\%: 61,27 e P: $8,93 \mathrm{mg} \mathrm{dm}{ }^{-3}$. Quanto aos micronutrientes: 19,59; 39,12; 10,$01 ; 1,44 \mathrm{mg} \mathrm{dm}^{-3}$ respectivamente para $\mathrm{Fe}, \mathrm{Mn}, \mathrm{Cu}$ e $\mathrm{Zn}$. Os teores de argila, silte e areia foram: 63,75; 17,50 e $18,75 \%$ respectivamente. A partir dos dados da análise de solo o mesmo foi corrigido e adubado.

Os tratamentos para os três experimentos consistiu na aplicação de quatro herbicidas em PPI (Experimento I), 20 herbicidas em PRÉ (Experimento II) e 25 herbicidas aplicados em PÓS (Experimento III) do cártamo, conforme descrito na Tabela 1.

Para avaliar a seletividade dos herbicidas, as sementes de cártamo foram semeadas a $2,0 \mathrm{~cm}$ de profundidade, anteriormente à aplicação dos tratamentos em PRÉ e para os tratamentos em PPI após a aplicação e incorporação dos herbicidas em solo revolvido até a profundidade de $10 \mathrm{~cm}$, para simular as condições de campo. Para os herbicidas em PÓS, as sementes foram alocadas a 2,0 cm de profundidade, e a aplicação foi realizada para plantas em estádio vegetativo de 3-4 folhas completamente desenvolvidas.

A aplicação dos herbicidas foi realizada com pulverizador costal pressurizado a $\mathrm{CO}_{2}$ com pressão constante de 2 bar, equipado com barra contendo 2 bicos do tipo leque Teejet XR 110.02, calibrado para gerar um volume de calda de $200 \mathrm{~L} \mathrm{ha}^{-1}$. As condições meteorológicas no momento da aplicação para o experimento I e II (PPI e PRÉ) foram: Temperatura de $26,0{ }^{\circ} \mathrm{C}$, umidade relativa do ar de $68 \%$ e velocidade do vento de $1,44 \mathrm{Km} \mathrm{h}^{-1}$. No experimento III, a aplicação foi realizada sob temperatura de $27,2{ }^{\circ} \mathrm{C}$, umidade relativa do ar de $56 \%$ e velocidade do vento de $0,66 \mathrm{Km} . \mathrm{h}^{-1}$. 
F. H. KRENCHINSKI et al.

Tabela 1. Herbicidas aplicados em pré-plantio incorporado, pré-plantio e pós-emergência na cultura do cártamo e seus respectivos nomes comerciais e doses.

\begin{tabular}{|c|c|c|c|}
\hline Nome Químico & Nome Comercial & Concentração & Dose (g i.a ha $\left.{ }^{-1}\right)$ \\
\hline \multicolumn{4}{|c|}{ Pré Plantio Incorporado } \\
\hline Controle & ---- & $\begin{array}{ll}--- \\
--\end{array}$ & $\begin{array}{l}--- \\
\end{array}$ \\
\hline Diclosulam & Spider WG & 840 g. $\mathrm{kg}^{-1}$ & 20 \\
\hline Metribuzin & Sencor SC & 480 g.L $\mathrm{L}^{-1}$ & 360 \\
\hline Pendimethalin & Herbadox EC & 400 g.L ${ }^{-1}$ & 1250 \\
\hline Trifluralin & Premerlin EC & 600 g.L. ${ }^{-1}$ & 1800 \\
\hline \multicolumn{4}{|c|}{ Pré Plantio } \\
\hline Controle & $\begin{array}{ll}--- \\
\end{array}$ & $\begin{array}{ll}--- \\
--1\end{array}$ & $\begin{array}{ll}--- \\
\end{array}$ \\
\hline $2,4-\mathrm{D}^{1}$ & DMA 806 BR & $670 \mathrm{~g} \cdot \mathrm{L}^{-1}$ & 335 \\
\hline Ametryn+clomazone & Sinerge EC & $300+200$ g. $L^{-1}$ & $900+600$ \\
\hline Atrazine & Primoleo SC & 400 & 2640 \\
\hline Carfentrazone & Aurora CE & 400 & 20 \\
\hline Carfentrazone + sulfrentrazone & Profit EC & $600+15$ & $11,25+450$ \\
\hline Clomazone & Gamit EC & 500 & 500 \\
\hline Ciclosulam & Spider WG & 840 g.kg ${ }^{-1}$ & 25 \\
\hline Flumioxazin & Sumisoya WP & 500 g.kg ${ }^{-1}$ & 20 \\
\hline Imazaqin $^{1}$ & Topgan CS & 150 & 2000 \\
\hline Imazethapyr $^{1}$ & Pivot SL & 100 & 80 \\
\hline Isoxaflutole & Provence $750 \mathrm{WG}$ & 750 g.kg ${ }^{-1}$ & 45 \\
\hline Lactofen & Cobra CE & 240 & 180 \\
\hline Mesotrione & Callisto SC & 480 & 240 \\
\hline Metribuzin & Sencor SC & 480 & 360 \\
\hline Metsulfuron & Ally WG & 600 g.kg ${ }^{-1}$ & 18 \\
\hline Pendimethalin & Herbadox EC & 400 & 1250 \\
\hline Saflufenacil & Heat WG & 700 g. $\mathrm{kg}^{-1}$ & 42 \\
\hline Metolachlor & Dual gold EC & 960 & 1200 \\
\hline Sulfrentrazone & Boral SC & 500 & 400 \\
\hline Trifluralin & Premerlin EC & 600 & 1800 \\
\hline
\end{tabular}

\footnotetext{
${ }^{1}$ dose em gramas de equivalente ácido por hectare $\left(\mathrm{g}\right.$ e.a.ha $\left.{ }^{-1}\right)$.
} 
F. H. KRENCHINSKI et al.

Tabela 1. Continuação.

\begin{tabular}{|c|c|c|c|}
\hline Nome Químico & Nome Comercial & Concentração & Dose (g i.a ha $\left.{ }^{-1}\right)$ \\
\hline \multicolumn{4}{|c|}{ Pós emergência da cultura } \\
\hline Controle & ------- & ------- & ------- \\
\hline Ametryn+clomazone & Sinerge EC & $300+200$ g. $L^{-1}$ & $900+600$ \\
\hline Atrazine & Primoleo SC & 400 & 2640 \\
\hline Bentazon & Basagran SL & 480 & 720 \\
\hline Bentazon + imazamox & Amplo SL & $600+28$ & $600+28$ \\
\hline Carfentrazone & Aurora CE & 400 & 20 \\
\hline Chlorimuron & Clorimuron Nortox WG & 250 g. $\mathrm{kg}^{-1}$ & 15 \\
\hline Clethodim + fenoxaprop & Podium S & $50+50$ & $40+40$ \\
\hline Clethodim & Select EC & 240 & 84 \\
\hline Clodinafop & Topik EC & 240 & 24 \\
\hline Fluroxypyr $^{1}$ & Starane EC & 200 & 100 \\
\hline Fluazifop+fomesafen & Fusiflex CS & $125+125$ & $200+200$ \\
\hline Fluazifop-P & Fusilade EW & 250 & 125 \\
\hline Flumiclorac & Radiant CE & 100 & 40 \\
\hline Flumioxazin & Sumisoya WP & 500 g. $\mathrm{kg}^{-1}$ & 20 \\
\hline Fluroxipir - meptilíco & Truper & & 230 \\
\hline Fomesafen & Flex SL & 870 & 225 \\
\hline Foramsulfuron + iodosulfuron & Equip Plus WG & $300+20$ & $36+24$ \\
\hline Haloxyfop $^{1}$ & Verdict R EC & 120 & 36 \\
\hline Imazethapyr $^{1}$ & Pivot SL & 100 & 80 \\
\hline Iodosulfuron & Hussar WG & 50 g.kg ${ }^{-1}$ & 3,5 \\
\hline Lactofen & Cobra CE & 240 & 150 \\
\hline Mesotrione & Callisto SC & 480 & 144 \\
\hline Nicosulfuron & Sanson SC & 40 & 50 \\
\hline Tembotrione & Soberan SC & 420 & 75,6 \\
\hline Tepraloxydim & Aramo EC & 200 & 70 \\
\hline
\end{tabular}

\footnotetext{
${ }^{1}$ dose em gramas de equivalente ácido por hectare $\left(\mathrm{g}\right.$ e.a.ha $\left.{ }^{-1}\right)$.
}

As variáveis analisadas foram fitotoxicidade a cultura aos 7, 14, 21 e 28 dias após a aplicação (DAA), utilizando a escala percentual de notas de $0-100$, na qual 0 representa ausência de injúrias visuais e, 100 representa a morte das plantas, (SBCPD, 1995). Além disso, foi avaliada a massa fresca e seca de plantas aos 35 DAA. A massa fresca foi pesada imediatamente após o corte das plantas em balança analítica, enquanto a massa seca foi determinada após secagem em estufa de circulação forçada de ar a $60^{\circ} \mathrm{C}$ até peso constante, com valores expressos em gramas.

Os resultados de controle, massa fresca e seca foram submetidos à análise de variância $(\mathrm{p} \leq 0,05)$ e, as médias dos tratamentos, foram comparadas pelo teste de Tukey $(\mathrm{p} \leq 0,05)$, utilizando-se o software Sisvar ${ }^{\circledR}$. 


\section{Resultados e discussão}

A aplicação de herbicidas em PPI na cultura do cártamo demonstrou que todos os herbicidas apresentaram sintomas de fitotoxicidade aos 7, 14, 21 e 28 DAA, com redução da massa fresca e seca para os herbicidas pendimethalin, diclosulam e metribuzin (Tabela 2). A fitotoxicidade causada pelos herbicidas pendimethalin, trifluralin e diclosulam foi baixa em relação a metribuzin, que apresentou fitotoxicidade de $40 \%$ aos 28 DAA e redução na massa fresca e seca superior a $74 \%$. Entretanto, os herbicida pendimethalin e diclosulam causaram redução superior a 37 e $44 \%$ na massa fresca e seca comparado ao controle sem aplicação de herbicida, respectivamente.

Tabela 2. Porcentagem de fitotoxicidade, massa fresca e massa seca de plantas de cártamo submetidas a aplicações de herbicidas em préplantio incorporado (PPI).

\begin{tabular}{ccccccc}
\hline \multirow{2}{*}{ Herbicidas } & \multicolumn{9}{c}{ Fitotoxicidade } & & Massa & Massa Seca \\
\cline { 2 - 4 } & 7 DAA & 14 DAA & 21 DAA & 28 DAA & Fresca & \\
\hline Controle & $0,0 \mathrm{a}$ & $0,0 \mathrm{a}$ & $0,0 \mathrm{a}$ & $0,0 \mathrm{a}$ & $26,5 \mathrm{a}$ & $1,5 \mathrm{a}$ \\
Diclosulam & $4,0 \mathrm{~b}$ & $1,7 \mathrm{a}$ & $2,5 \mathrm{a}$ & $4,7 \mathrm{a}$ & $13,8 \mathrm{~b}$ & $0,9 \mathrm{~b}$ \\
Metribuzin & $6,0 \mathrm{c}$ & $26,2 \mathrm{~b}$ & $32,0 \mathrm{~b}$ & $40,5 \mathrm{~b}$ & $3,0 \mathrm{c}$ & $0,4 \mathrm{c}$ \\
Pendimethalin & $2,5 \mathrm{~b}$ & $4,0 \mathrm{a}$ & $2,7 \mathrm{a}$ & $4,5 \mathrm{a}$ & $14,8 \mathrm{~b}$ & $0,9 \mathrm{cb}$ \\
Trifluralin & $3,7 \mathrm{~b}$ & $5,0 \mathrm{a}$ & $4,5 \mathrm{a}$ & $3,7 \mathrm{a}$ & $25,9 \mathrm{a}$ & $1,6 \mathrm{a}$ \\
\hline Média & 3,2 & 7,4 & 8,3 & 10,7 & 16,8 & 1,1 \\
\hline DMS & 1,5 & 7,4 & 7,1 & 5,4 & 5,0 & 0,5 \\
\hline CV\% & 12,1 & 15,9 & 18,9 & 13,1 & 13,7 & 12,0 \\
\hline
\end{tabular}

Letras minúsculas iguais na coluna não diferem entre si pelo teste de Tukey a 5\% de probabilidade.

Para aplicação em PPI, o herbicida trifluralin foi o único que não reduziu significativamente a massa fresca e seca das plantas de cártamo em relação ao controle sem aplicação, com fitotoxicidade inferior a $5 \%$ ao longo do período avaliado (Tabela 2). Resultados similares foram encontrados para o crambe onde o herbicida metribuzin aplicado em PPI não foi seletivo, causando a morte das plantas aos 28 DAA enquanto que o herbicida trifluralin foi o único seletivo para a cultura (SOUZA et al. 2014). No entanto, a aplicação de trifluralin na cultura da manona causou fitotoxicidade superior a $15 \%$ nas plantas e redução da produtividade, não sendo considerada opção para manejo de plantas daninhas (MACIEL et al. 2007).

Para os herbicidas aplicados em PRÉ, somente 4 foram considerados seletivos para as plantas de cártamo (Tabela 3). Elevada fitotoxicidade com níveis superiores a $70 \%$ foram observados para os herbicidas ametryn + clomazone, carfentrazone + sulfrentrazone, clomazone, mesotrione, e saflufenacil aos 7 DAA. Injúria inferior a 10\% foi evidenciado para os herbicidas 2,4-D, atrazine, carfentrazone, flumioxazin, imazethapyr, metribuzin, pendimethalin e trifluralin aos 7 DAA. Para as avaliações realizadas aos 14 e 21 DAA, houve aumento da fitotoxicidade para todos os tratamentos aplicados em PRÉ, exceto para 2,4-D, carfentrazone, flumioxazin e trifluralin onde houve redução da injúria (Tabela 3 ).

Os herbicidas ametryn+clomazone, carfentrazone + sulfrentrazone, clomazone, mesotrione e saflufenacil ocasionaram morte total das plantas de cártamo aos 28 DAA, enquanto que a aplicação de 2,4-D, carfentrazone, flumioxazin, trifluralin e pendimethalin resultou baixa fitotoxicidade, sendo considerados seletivos ao cártamo (Tabela 3). Para cultura do girassol, os herbicidas clomazone e imazethapyr também não foram seletivos, causando redução na produção de aquênios (MASCARENHAS et al. 2012; VIDAL; FLECK, 1994).

As maiores produções de massa fresca e seca foram com os herbicidas 2,4-D, carfentrazone, flumioxazin e trifluralin, não diferindo significativamente do controle sem a aplicação de herbicidas (Tabela 3). Alguns herbicidas como, atrazine e pendimethalin apresentaram menor sintomas de fitotoxicidade (Tabela 3), porém causaram redução da massa fresca e seca comparado ao controle e aos herbicidas considerados seletivos. Os demais ingredientes ativos testados provocaram elevada redução de massa fresca e seca. 
F. H. KRENCHINSKI et al.

Tabela 3. Porcentagem de fitotoxicidade, massa fresca e massa seca de plantas de cártamo submetidas a aplicações de herbicidas em préemergência (PRÉ).

\begin{tabular}{|c|c|c|c|c|c|c|}
\hline \multirow{2}{*}{ Herbicidas } & \multicolumn{4}{|c|}{ Fitotoxicidade } & \multirow{2}{*}{$\begin{array}{l}\text { Massa } \\
\text { Fresca }\end{array}$} & \multirow{2}{*}{ Massa Seca } \\
\hline & 7 DAA & $14 \mathrm{DAA}$ & $21 \mathrm{DAA}$ & $28 \mathrm{DAA}$ & & \\
\hline Controle & $0,0 \mathrm{a}$ & $0,0 \mathrm{a}$ & $0,0 \mathrm{a}$ & $0,0 \mathrm{a}$ & $31,8 \mathrm{a}$ & $2,5 \mathrm{a}$ \\
\hline $2,4-\mathrm{D}$ & 5,7 abcd & $4,2 \mathrm{a}$ & $2,0 \mathrm{a}$ & $0,0 \mathrm{a}$ & $29,7 \mathrm{a}$ & $2,3 \mathrm{a}$ \\
\hline Ametryn+clom, ${ }^{2}$ & 95,01 & $98,7 \mathrm{f}$ & $98,7 \mathrm{e}$ & $100,0 \mathrm{f}$ & $0,0 \mathrm{f}$ & $0,0 \mathrm{~d}$ \\
\hline Atrazine & $4,5 \mathrm{abc}$ & $8,0 \mathrm{a}$ & $6,7 \mathrm{a}$ & $6,2 \mathrm{a}$ & $13,7 \mathrm{~b}$ & $1,0 \mathrm{bc}$ \\
\hline Carf, + sulf, $^{3}$ & 93,71 & $95,2 \mathrm{f}$ & $96,5 \mathrm{e}$ & $100,0 \mathrm{f}$ & $0,0 \mathrm{f}$ & $0,0 \mathrm{~d}$ \\
\hline Carfentrazone ${ }^{1}$ & 9,0 bcdef & $0,0 \mathrm{a}$ & $0,0 \mathrm{a}$ & $0,0 \mathrm{a}$ & $32,2 \mathrm{a}$ & $2,7 \mathrm{a}$ \\
\hline Clomazone & 95,01 & $96,2 \mathrm{f}$ & $96,2 \mathrm{e}$ & $100,0 \mathrm{f}$ & $0,0 \mathrm{f}$ & $0,0 \mathrm{~d}$ \\
\hline Diclosulam & 12,7 ef & $42,5 \mathrm{bc}$ & $55,7 \mathrm{bcd}$ & $86,0 \mathrm{de}$ & $1,1 \mathrm{f}$ & $0,3 \mathrm{~cd}$ \\
\hline Flumioxazin & $3,7 \mathrm{abc}$ & $3,2 \mathrm{a}$ & $1,2 \mathrm{a}$ & $0,5 \mathrm{a}$ & $30,4 \mathrm{a}$ & $2,4 \mathrm{a}$ \\
\hline Imazaqin & $12,5 \mathrm{def}$ & $46,5 \mathrm{bc}$ & $52,5 \mathrm{bcd}$ & $67,0 \mathrm{c}$ & 1,7 ef & $0,3 \mathrm{~cd}$ \\
\hline Imazethapyr & 6,0 abcde & $41,0 \mathrm{bc}$ & $55,5 \mathrm{bcd}$ & $79,0 \mathrm{~d}$ & $0,8 \mathrm{f}$ & $0,4 \mathrm{~cd}$ \\
\hline Isoxaflutole & $35,5 \mathrm{i}$ & $38,0 \mathrm{bc}$ & $43,0 \mathrm{~b}$ & $58,5 \mathrm{c}$ & $6,1 \mathrm{~cd}$ & $0,5 \mathrm{bcd}$ \\
\hline Lactofen & $21,5 \mathrm{gh}$ & $61,2 \mathrm{~d}$ & $60,5 \mathrm{~cd}$ & $56,5 \mathrm{c}$ & $9,0 \mathrm{c}$ & $0,8 \mathrm{bcd}$ \\
\hline Mesotrione & $83,0 \mathrm{k}$ & $96,0 \mathrm{f}$ & 97,7 e & $100,0 \mathrm{f}$ & $0,0 \mathrm{f}$ & $0,0 \mathrm{~d}$ \\
\hline Metribuzin & $9,5 \mathrm{cdef}$ & 80,7 e & 89,0 e & 96,0 ef & $0,5 \mathrm{f}$ & $0,1 \mathrm{~d}$ \\
\hline Metsulfuron $^{4}$ & $15,5 \mathrm{fg}$ & $36,2 \mathrm{~b}$ & $46,0 \mathrm{bc}$ & $62,5 \mathrm{c}$ & $5,3 \mathrm{~d}$ & $0,5 \mathrm{bcd}$ \\
\hline Pendimethalin & $2,5 \mathrm{ab}$ & $8,5 \mathrm{a}$ & $6,2 \mathrm{a}$ & $3,0 \mathrm{a}$ & $15,1 \mathrm{~b}$ & $1,3 \mathrm{~b}$ \\
\hline Saflufenacil & $70,0 \mathrm{j}$ & $96,7 \mathrm{f}$ & 97,2 e & $100,0 \mathrm{f}$ & $0,0 \mathrm{f}$ & $0,0 \mathrm{~d}$ \\
\hline S-metolachlor & $27,7 \mathrm{~h}$ & $41,5 \mathrm{bc}$ & $41,0 \mathrm{~b}$ & $27,5 \mathrm{~b}$ & $5,9 \mathrm{~cd}$ & $0,6 \mathrm{bcd}$ \\
\hline Sulfrentrazone & $40,2 \mathrm{i}$ & $49,5 \mathrm{~cd}$ & $62,5 \mathrm{~d}$ & $61,5 \mathrm{c}$ & $4,7 \mathrm{de}$ & $0,4 \mathrm{bcd}$ \\
\hline Trifluralin & 6,0 abcde & $5,00 \mathrm{a}$ & $4,25 \mathrm{a}$ & $3,00 \mathrm{a}$ & $30,71 \mathrm{a}$ & $2,47 \mathrm{a}$ \\
\hline Média & 30,9 & 45,2 & 48,2 & 52,7 & 10,4 & 0,9 \\
\hline DMS & 6,8 & 12,0 & 15,0 & 11,1 & 3,3 & 0,9 \\
\hline $\mathrm{CV} \%$ & 8,3 & 10,0 & 11,8 & 7,9 & 12,0 & 17,5 \\
\hline
\end{tabular}

Letras minúsculas iguais na coluna não diferem entre si pelo teste de Tukey a 5\% de probabilidade, ${ }^{1}$ carfentrazone - ethyl; ${ }^{2}$ ametryn+clomazone; ${ }^{3}$ carfentrazone - ethyl + sulfrentrazone; ${ }^{4}$ metsulfuron.

$\mathrm{O}$ herbicida trifluralin apresentou-se seletivo à cultura do cártamo, em ambas as modalidades de aplicação (PPI e PRÉ), podendo ser uma ferramenta bastante válida no controle de plantas daninhas nessa cultura (Tabelas 2 e 3), assim como nas culturas de girassol (MASCARENHAS et al. 2012), mamona (MEDEIROS et al. 2013), e crambe (SOUZA et al. 2014), dependendo da textura do solo. Esta molécula pertence ao grupo químico das dinitroanilinas e controla eficientemente plantas daninhas gramíneas e algumas espécies dicotiledôneas com sementes de menor tamanho (RAIMONDI et al. 2011; SOFIATTI et al. 2012). $\mathrm{O}$ modo de ação envolve a inibição da polarização da tubulina e interrupção da ocorrência de mitose nas células (SCHIBLER; HUANG, 1991). A seletividade do trifluralin 
pode ocorrer devido a insensibilidade do local de ação, metabolização diferencial ou por diferença de posicionamento do herbicida em relação ao banco de sementes (VIDAL; FLECK, 2001). Segundo Kalsing e Vidal (2013), para experimentos conduzidos em vasos, o perfil do solo passa a não ser fator de seletividade, portanto, as prováveis causas da seletividade são à insensibilidade do local de ação e/ou metabolização diferencial.

Os herbicidas diclosulam, metribuzin e pendimethalin aplicados em PPI e PRÉ não apresentaram seletividade ao cártamo. Para a mistura comercial de carfentrazone + sulfrentrazone, os resultados não evidenciaram seletividade, exceto para aplicação isolada de carfentrazone, porém esse aspecto pode ser explicado pela não seletividade do herbicida sulfrentrazone quando aplicado de forma isolada, o qual ocasionou baixa produção de massa fresca $(4,75 \mathrm{~g})$ (Tabela 3).

Para aplicação em PÓS, os herbicidas ametryn+clomazone, atrazine, bentazon, bentazon + imazamox, lactofen, carfentrazone, chlorimuron, fluazifop$\mathrm{P}+$ fomesafen e fomesafen apresentaram elevada injúria visual aos 7 DAA (Tabela 4). Todavia, injúrias inferiores a $5 \%$ foram observadas para os herbicidas clethodim + fenoxaprop e clodinafop aos 7 DAA. Aos 14 DAA, alguns herbicidas apresentaram redução, como o cletodim (11\%), e outros aumentaram a fitotoxicidade, como o lactofen e basagran (100\%) (Tabela 4), Os graminicidas, de modo geral, apresentaram as menores injúrias, porém ao serem testados em mamona (Ricinus communis), não ocasionaram injúrias visuais, mas sim reduções de área foliar, provavelmente devido a fatores intrínsecos à cultura (SOFIATTI et al. 2008).

Tabela 4. Porcentagem de fitotoxicidade, massa fresca e massa seca de plantas de cártamo submetidas a aplicações de herbicidas em pósemergência (PÓS).

\begin{tabular}{|c|c|c|c|c|c|c|}
\hline \multirow{2}{*}{ Herbicidas } & \multicolumn{4}{|c|}{ Fitotoxicidade } & \multirow{2}{*}{$\begin{array}{l}\text { Massa } \\
\text { Fresca }\end{array}$} & \multirow{2}{*}{ Massa Seca } \\
\hline & $7 \mathrm{DAA}$ & $14 \mathrm{DAA}$ & $21 \mathrm{DAA}$ & $28 \mathrm{DAA}$ & & \\
\hline Controle & $0,0 \mathrm{a}$ & $0,0 \mathrm{a}$ & $0,0 \mathrm{a}$ & $0,0 \mathrm{a}$ & $46,1 \mathrm{a}$ & $4,7 \mathrm{a}$ \\
\hline Ametryn+clomazone & 100,01 & $100,0 \mathrm{~g}$ & $100,0 \mathrm{i}$ & $100,0 \mathrm{~g}$ & $0,0 \mathrm{~d}$ & $0,0 \mathrm{e}$ \\
\hline Atrazine & 100,01 & $100,0 \mathrm{~g}$ & $100,0 \mathrm{i}$ & $100,0 \mathrm{~g}$ & $0,0 \mathrm{~d}$ & $0,0 \mathrm{e}$ \\
\hline Bentazon & 100,01 & $100,0 \mathrm{~g}$ & $100,0 \mathrm{i}$ & $100,0 \mathrm{~g}$ & $0,0 \mathrm{~d}$ & $0,0 \mathrm{e}$ \\
\hline Bentazon + imazamox & 100,01 & $100,0 \mathrm{~g}$ & $100,0 \mathrm{i}$ & $100,0 \mathrm{~g}$ & $0,0 \mathrm{~d}$ & $0,0 \mathrm{e}$ \\
\hline Carfentrazone - ethyl & $81,0 \mathrm{ijk}$ & 84,0 efg & $60,0 \mathrm{de}$ & $34,5 \mathrm{f}$ & $25,5 \mathrm{~b}$ & $2,1 \mathrm{bcd}$ \\
\hline Chlorimuron - ethyl & $81,0 \mathrm{ijk}$ & $75,0 \mathrm{def}$ & $83,5 \mathrm{fgh}$ & $100,0 \mathrm{~g}$ & $0,0 \mathrm{~d}$ & $0,0 \mathrm{e}$ \\
\hline Clethodim + Fenoxaprop & $4,0 \mathrm{ab}$ & $97,7 \mathrm{~g}$ & $98,0 \mathrm{hi}$ & $100,0 \mathrm{~g}$ & $8,8 \mathrm{c}$ & 0,9 cde \\
\hline Cletodim & $13,5 \mathrm{ab}$ & $11,0 \mathrm{a}$ & $9,7 \mathrm{ab}$ & $8,0 \mathrm{ab}$ & $45,6 \mathrm{a}$ & $4,4 \mathrm{a}$ \\
\hline Clodinafop & $4,5 \mathrm{ab}$ & $93,0 \mathrm{fg}$ & $81,2 \mathrm{fg}$ & $29,0 \mathrm{ef}$ & $26,4 \mathrm{~b}$ & $2,3 \mathrm{~d}$ \\
\hline Floroxypyr & 53,5 efg & $57,0 \mathrm{bcd}$ & $77,2 \mathrm{fg}$ & $100,0 \mathrm{~g}$ & $0,0 \mathrm{~d}$ & $0,0 \mathrm{e}$ \\
\hline Fluazifop+fomesafen & $86,0 \mathrm{jkl}$ & $91,0 \mathrm{fg}$ & 90,5 ghi & $94,0 \mathrm{~g}$ & $4,3 \mathrm{~cd}$ & $0,8 \mathrm{de}$ \\
\hline Fluazifop-p-butyl & $17,2 \mathrm{bc}$ & $15,0 \mathrm{a}$ & $12,5 \mathrm{ab}$ & $6,0 \mathrm{ab}$ & $47,1 \mathrm{a}$ & $4,5 \mathrm{a}$ \\
\hline Flumiclorac-pentyl & $58,0 \mathrm{fgh}$ & $50,5 \mathrm{bc}$ & $30,0 \mathrm{c}$ & $18,0 \mathrm{~cd}$ & $27,1 \mathrm{~b}$ & $2,6 \mathrm{~b}$ \\
\hline Flumioxazin & $65,5 \mathrm{ghi}$ & $62,5 \mathrm{bcd}$ & $54,0 \mathrm{~d}$ & $38,0 \mathrm{f}$ & $23,3 \mathrm{~b}$ & $2,3 \mathrm{bc}$ \\
\hline Fluroxipir - meptilíco & $63,0 \mathrm{fgh}$ & $92,0 \mathrm{fg}$ & 98,5 hi & $100,0 \mathrm{~g}$ & $0,0 \mathrm{~d}$ & $0,0 \mathrm{e}$ \\
\hline Fomesafen & $93,5 \mathrm{kl}$ & $95,0 \mathrm{~g}$ & $97,7 \mathrm{hi}$ & $100,0 \mathrm{~g}$ & $0,0 \mathrm{~d}$ & $0,0 \mathrm{e}$ \\
\hline Foramsulfuron + iodosulfuron & $63,5 \mathrm{fgh}$ & $63,5 \mathrm{bcd}$ & 86,0 fghi & $100,0 \mathrm{~g}$ & $0,0 \mathrm{~d}$ & $0,0 \mathrm{e}$ \\
\hline Haloxifope-p-metílico & $18,2 \mathrm{bc}$ & $17,2 \mathrm{a}$ & $15,0 \mathrm{abc}$ & $11,2 \mathrm{bc}$ & $47,4 \mathrm{a}$ & $4,5 \mathrm{a}$ \\
\hline Imazethapyr & 71,5 hij & $68,5 \mathrm{cde}$ & $80,5 \mathrm{fg}$ & $100,0 \mathrm{~g}$ & $0,0 \mathrm{~d}$ & $0,0 \mathrm{e}$ \\
\hline Iodosulfuron-methyl & 49,0 def & $50,0 \mathrm{bc}$ & 84,5fghi & $100,0 \mathrm{~g}$ & $0,0 \mathrm{~d}$ & $0,0 \mathrm{e}$ \\
\hline
\end{tabular}

Letras minúsculas iguais na coluna não diferem entre si pelo teste de Tukey a $5 \%$ de probabilidade

Rev. Bras. Herb., v. 18, n. 1, e. 636, jan./mar., 2019 
F. H. KRENCHINSKI et al.

Tabela 4. Continuação.

\begin{tabular}{|c|c|c|c|c|c|c|}
\hline \multirow{2}{*}{ Herbicidas } & \multicolumn{4}{|c|}{ Fitotoxicidade } & \multirow{2}{*}{$\begin{array}{l}\text { Massa } \\
\text { Fresca }\end{array}$} & \multirow{2}{*}{ Massa Seca } \\
\hline & $7 \mathrm{DAA}$ & 14 DAA & $21 \mathrm{DAA}$ & $28 \mathrm{DAA}$ & & \\
\hline Lactofen & 100,01 & $100,0 \mathrm{~g}$ & $100,0 \mathrm{i}$ & $100,0 \mathrm{~g}$ & $0,0 \mathrm{~d}$ & $0,0 \mathrm{e}$ \\
\hline Mesotrione & $39,7 \mathrm{de}$ & $62,0 \mathrm{bcd}$ & $100,0 \mathrm{i}$ & $100,0 \mathrm{~g}$ & $0,0 \mathrm{~d}$ & $0,0 \mathrm{e}$ \\
\hline Nicosulfuron & 66,5 ghi & $48,5 \mathrm{~b}$ & 72,0 ef & $100,0 \mathrm{~g}$ & $0,0 \mathrm{~d}$ & $0,0 \mathrm{e}$ \\
\hline Tembotrione & $33,2 \mathrm{~cd}$ & $53,0 \mathrm{bc}$ & 74,0 ef & $100,0 \mathrm{~g}$ & $2,3 \mathrm{dc}$ & $0,7 \mathrm{e}$ \\
\hline Tepraloxydim & $15,5 \mathrm{ab}$ & $14,5 \mathrm{a}$ & $24,0 \mathrm{bc}$ & $22,5 \mathrm{de}$ & $28,5 \mathrm{~b}$ & $2,7 \mathrm{~b}$ \\
\hline Média & 56,8 & 65,4 & 70,3 & 71,6 & 13,5 & 1,3 \\
\hline DMS & 16,2 & 19,5 & 16,1 & 10,0 & 6,5 & 1,4 \\
\hline $\mathrm{CV} \%$ & 10,5 & 11,1 & 8,5 & 5,2 & 17,8 & 18,3 \\
\hline
\end{tabular}

Letras minúsculas iguais na coluna não diferem entre si pelo teste de Tukey a $5 \%$ de probabilidade

Aos 21 e 28 DAA, a maioria dos herbicidas (16 ativos) ocasionaram morte total das plantas e outros apresentaram maiores teores de fitotoxicidade, como o fluazifop+fomesafen (Tabela 4). Entretanto, os herbicidas carfentrazone, clethodim, clodinafop, fluazifop-P, flumiclorac, flumioxazin, haloxyfop e tepraloxydim, apresentaram redução da injúria visual aos 21 e 28 DAA (Tabela 4), ressaltando o possível efeito do metabolismo diferencial da planta, formando assim compostos não tóxicos e também pela recuperação ao longo do seu ciclo. De maneira similar, a aplicação em pós-emergência de nicosulfuron, chlorimuron e imazethapyr acarretou em alta fitotoxicidade sobre duas variedades de girassol, não afetando o teor de clorofila, porém a produtividade foi diminuída em relação à testemunha sem aplicação (BRIGHENTI, 2012; MASCARENHAS et al. 2012).

O acúmulo de massa fresca não diferiu estatisticamente da testemunha sem aplicação para os herbicidas clethodim, fluazifop e haloxyfop, demonstrando que a baixa fitotoxicidade destes herbicidas possibilita sua utilização em plantas de cártamo, sem ocasionar danos significativos ao desenvolvimento.

Os herbicidas clethodim, fluazifop-P e haloxyfop atuam na inibição da enzima ACCase, sendo importantes ferramentas no controle de plantas daninhas monocotiledôneas em culturas dicotiledôneas (JASPER et al. 2015). Vale destacar que, alguns herbicidas inibidores da ACCase, não foram seletivos para o cártamo (clodinafop e tepraloxydim), mesmo causando menor injúria visual em comparação a alguns outros pertencentes ao mecanismo de ação de inibição da ALS (Tabela 4). Maciel et al. (2011), avaliando a seletividade de herbicidas inibidores de ACCase na mamoneira evidenciaram que o tepraloxydim na dose de 400 g i.a. ha ${ }^{-1}$ causou pequena injúria visual, sem redução da produtividade. Essa diferença pode ser atribuída pela diferença entre espécies e a sensibilidade, ou habilidade de cada uma em metabolizar estes herbicidas (MARTINS et al. 2007).

Convém destacar que o cártamo é uma cultura sensível a aplicação de herbicidas comparado a outras culturas comerciais, haja visto que todos os herbicidas aplicados causaram injúria visual pelo menos aos 7 DAA, nas três modalidades de aplicação (PPI, PRÉ e PÓS), mesmo aqueles herbicidas que ao final foram considerados seletivos para a cultura.

\section{Conclusões}

O herbicida trifluralin foi o único a apresentar seletividade ao cártamo em PPI, assim como elevado potencial de uso em PRÉ. Os herbicidas 2,4-D, carfentrazone, e flumioxazin foram seletivos em PRÉ, enquanto que clethodim, fluazifop$\mathrm{P}$ e haloxyfop apresentam maior seletividade para aplicação em PÓS.

\section{Referências}

Bagheri, H.; Sam-Daliri, M. Effect of water stress on agronomic traits of spring safflower cultivars (Carthamus tinctorius L.). Australian Journal of Basic and Applied Sciences, v.5, n.12, p.2621-2624, 2011.

Brighenti, A.M.; Resistência do girassol a herbicidas inibidores da enzima acetolactatosintase. Pesquisa Agropecuária Tropical, v.42, n.2, p.225-230, 2012.

De Oliveira Neto A.M.; Guerra, N. Maciel, C.D de G. Da Silva, T.R.B. Lima, G.G. de R. Seletividade de herbicidas aplicados em pré-emergência na cultura do crambe. Revista Brasileira de Herbicidas. v.10, n.1, p.49-56, 2011.

FAOSTAT - Food And Agriculture Organization Of The United Nations. Crops. 2019. Disponível em:Acesso em: 28 mar. 2019.

Grigolli J.F.J.; Selectivity of post-emergence herbicides to crambe1. Revista Brasileira de Herbicidas. v.14, n.1, p.30- 


\section{$37,2015$.}

Hammer, K.; Heller, J. Promoting the conservation and use of underutilized and neglected crops. Internacional Plant Genetic Resources Institute, v.8, p.223-227, 1998.

Harker, K.N.; O'Donovan, J.T. Recent Weed Control, Weed Management, and Integrated Weed Management. Weed Technology, v.27, p.1-11, 2013.

Jasper, S.P.; Velini, E.D.; Rossetto, M.R.M.; Carbonari, C.A.; Trindade, M.L.B. Aplicação em subdose do haloxyfop -methyl na aveia-preta. Ciência Rural, v.45, n.4, p.637-643, 2015.

Kalsing, A.; Vidal, R.A. Seletividade de herbicidas residuais ao feijão-comum durante o período inicial da fase vegetativa. Planta daninha.v.31, n.2, p.411-417, 2013.

Maciel, C.D.G.; Poletine, J.P. Velini, E.D. Zanotto, M.D. Amaral, J.G. C. Santos, H.R. Artioli, J.C. et al. Seletividade de herbicidas em cultivares de mamona. Revista Brasileira de Oleaginosas e Fibrosas, v. 11, n. 1, p.47-54, 2007.

Maciel, C.D.G.; Silva, T.R.B. Poletine, J.P. Velini, E.D. Zanotto, M.D. Martins, F.M. Gava, F. Seletividade e eficácia de herbicidas inibidores da enzima ACCase na cultura da mamona. Planta Daninha, v.29, n.3, p.609-616, 2011.

Marques, R.F. Períodos de Interferência de Plantas Daninhas e Seletividade a Herbicidas em Canola, Niger e Cártamo. 2017. 79 f. Tese (Doutorado em Agronomia) Faculdade de Ciências Agrárias, Universidade Federal da Grande Dourados, Dourados, 2017.

Martins, D.; Triguero, L.R.C. Domingos, V.D. Martins, C.C. Marchi, S.R. de. Costa, N.V. da. Seletividade de herbicidas aplicados em pós-emergência sobre capim-braquiária. Revista Brasileira de Zootecnia. v.36, n.6, p.1969-1974, 2007.

Mascarenhas, M.H.T.; Karam, D. Lara, J.F.R. Seletividade de herbicidas e dinâmica populacional de plantas daninhas na cultura do girassol para a produção de biodiesel. Revista Brasileira de Herbicidas, v. 11, n. 2, p. 174-186, 2012.

Medeiros, Katty AA de L.; Solfiatti, V. Silva, H. Zonta, J.H. Costa, A.G.F. Silva, J.B. Tolerância da mamoneira ao herbicida trifluralin em solos com diferentes texturas. Revista Brasileira de Engenharia Agricola e Ambiental-Agriambi, v. 17, n. 12, p.1333-1339, 2013.

Negrisoli, E.; Velini, E.D. Tofoli, G.R. Cavenaghi, A.L. Martins, D. Morelli, J.L. Costa, A.G.F. Seletividade de herbicidas aplicados em pré-emergência na cultura de canade-açúcar tratada com nematicidas. Planta Daninha, v.22, n.4, p.567-575, 2004.

Nepomuceno, M.; Alves, P.L.C.A. Dias, T.C.S. Pavani, M.C.M.D. Períodos de interferência das plantas daninhas na cultura da soja nos sistemas de semeadura direta e convencional. Planta Daninha, v.25, n.1, p.43-50, 2007.

Rahamatalla, A.B.; Babiker, E.E. Krishna, A.G. El Tinay, A.H. Changes in fatty acids composition during seed growth and physicochemical characteristics of oil extracted from four safflower cultivars. Plant Foods for Human Nutrition, v. 56, n. 4, p. 385-395, 2001.

Raimondi, M.A.; Oliveira Jr, R.S. Constantin, J. Biffe, D.F. Arantes, J.G.Z. Franchini, L.H et al. Atividade residual de herbicidas aplicados ao solo em relação ao controle de quatro espécies de Amaranthus. Planta Daninha, v. 28, n. 5, p.1073-1085, 2011.

SBCPD. Procedimentos para instalação, avaliação e análise de experimentos com herbicidas, Londrina: 1995. $42 p$.

Schibler, M.J.; Huang, B. The colR4 and colR15 betatubulin mutations in Chlamydomonas reinhardtii confer altered sensitivities to microtubule inhibitors and herbicides by enhancing microtubule stability. The Journal of cell biology, v. 113, n. 3, p. 605-614, 1991.

Silva, A.A.; Silva, J.F. Tópicos em manejo de plantas daninhas. Viçosa: Universidade Federal de Viçosa, 2007. p. 17-61.

Sofiatti, V.; Silva, D.M.A.; Severino, L.S.; SILVA, F.D.O.; Cardoso, G.D.; FREIRE, M.D.O.; Sampaio, L.R. Seletividade de herbicidas pós-emergentes à cultura da mamoneira. In: Congresso Brasileiro de Mamona, 3., 2008, Salvador. Anais... Salvador: Energia e Ricinoquímica: SEAGRI: Embrapa Algodão, 2008.

Sofiatti, V.; Severino, L.V. Silva, F.M.O. Silva, V.N.B. Britto, G.G. Pre and postemergence herbicides for weed control in castor crop. Industrial Crops and Products, v.37, n.1, p.235-237, 2012.

Souza, F.; Sasso, G. Vitorino, H dos S. Fioreze, A.C da C.L. Pereira, M.R.C. Martins, D. Seletividade de herbicidas na cultura de crambe. Semina-ciencias Agrarias, v.35, n.1, p. 161-168, 2014.

Vidal, R.A.; Fleck, N.G. Injúria potencial de herbicidas ao solo ao girassol: IV-rendimento de aquênios e componentes do rendimento. Planta Daninha. v.12, n.1, p. 44-51, 1994.

Vidal R.A., Fleck N.G. Inibidores de crescimento da parte aérea. In: Herbicidologia. Vidal R.A., Merotto JR. A. Editores. Porto Alegre: Evangraf, 2001.p. 123-130. 